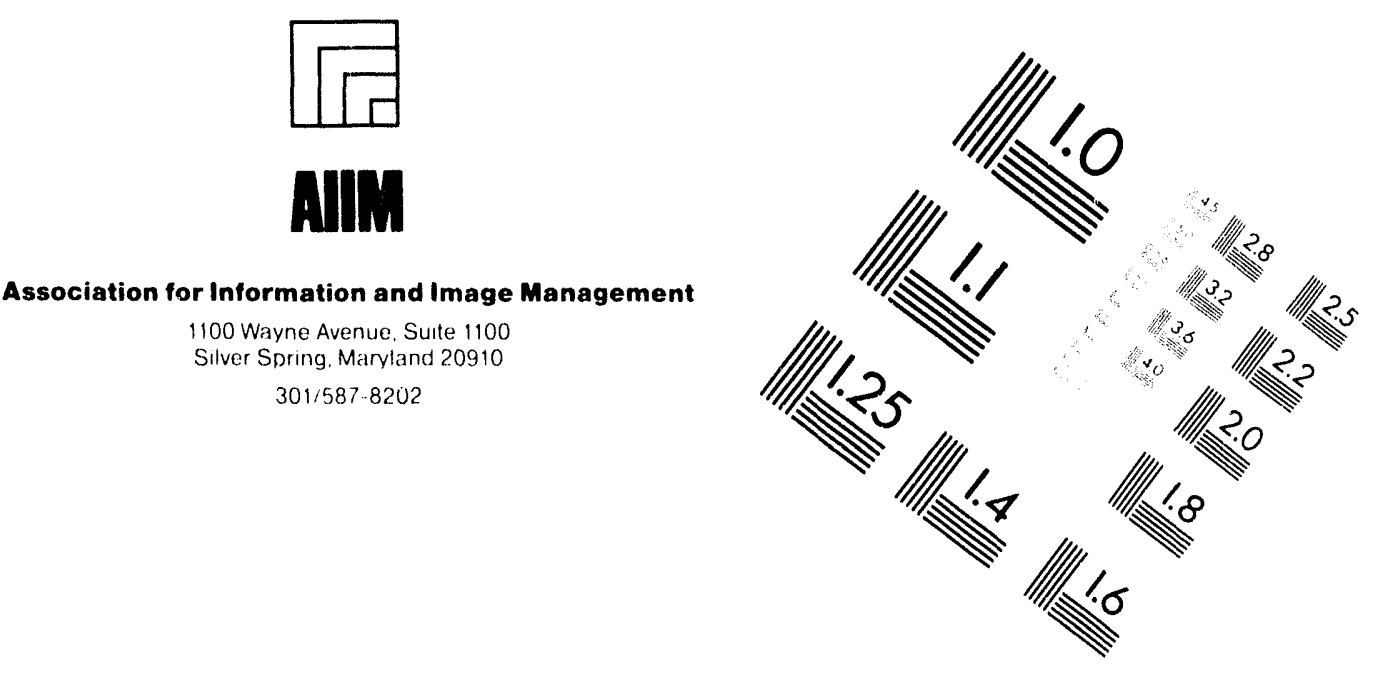

\title{
Centimeter
}

$\begin{array}{llllllllllllllll}1 & 2 & 3 & 4 & 5 & 6 & 7 & 8 & 9 & 10 & 11 & 12 & 13 & 14 & 15 & \mathrm{~mm}\end{array}$

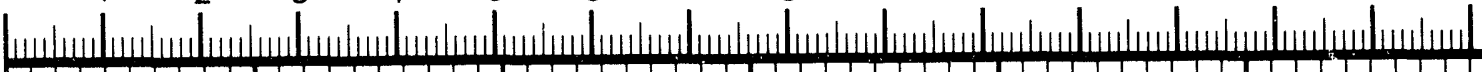

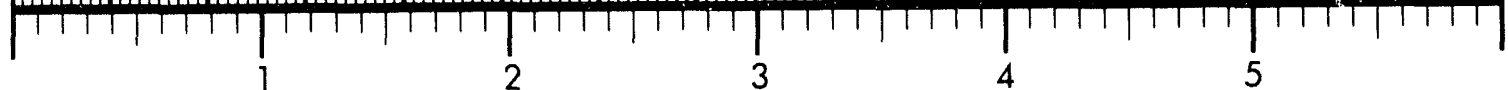
Inches
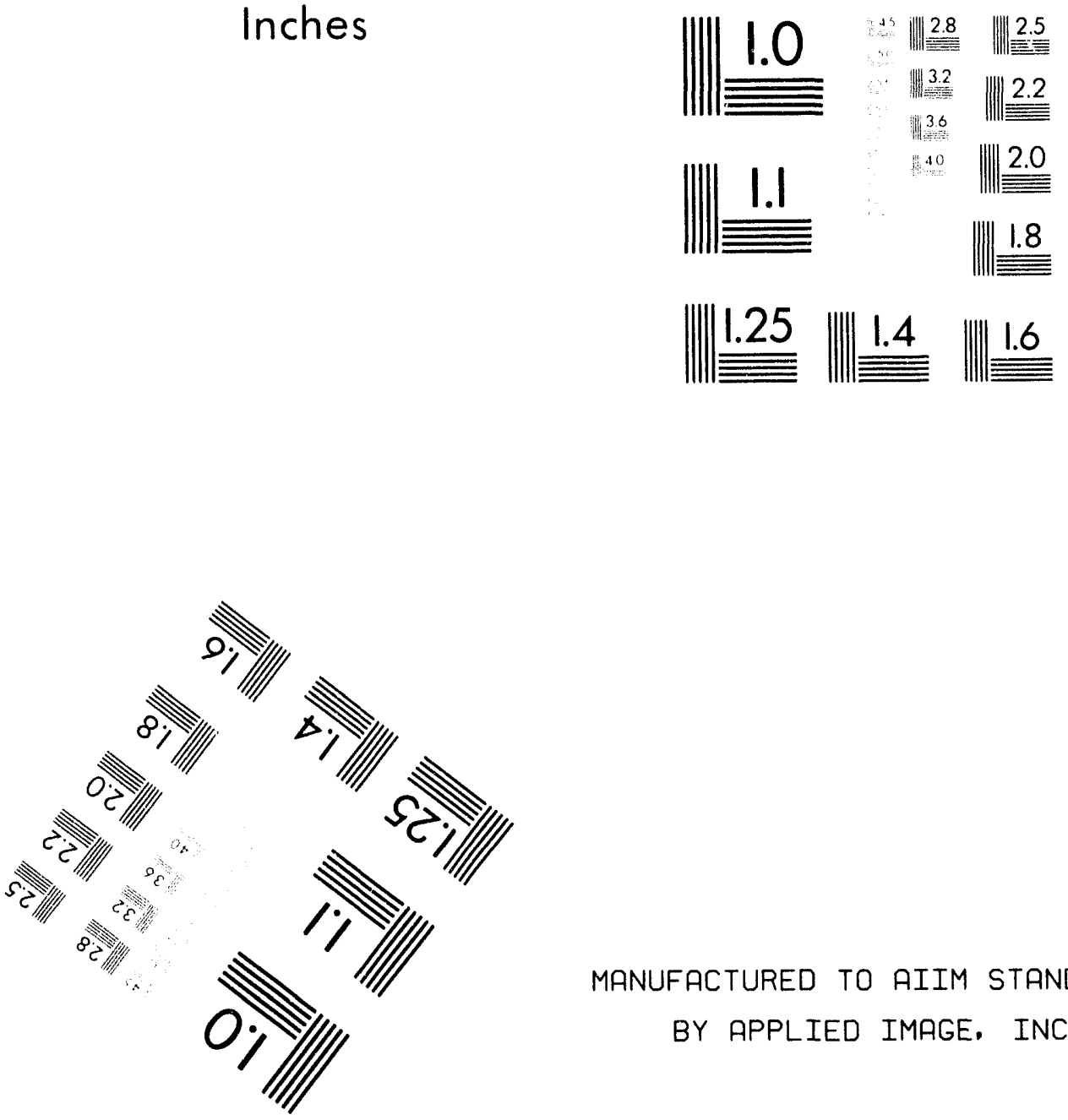

MANUFACTURED TO AIIM STANDARDS

BY APPLIED IMAGE, INC.

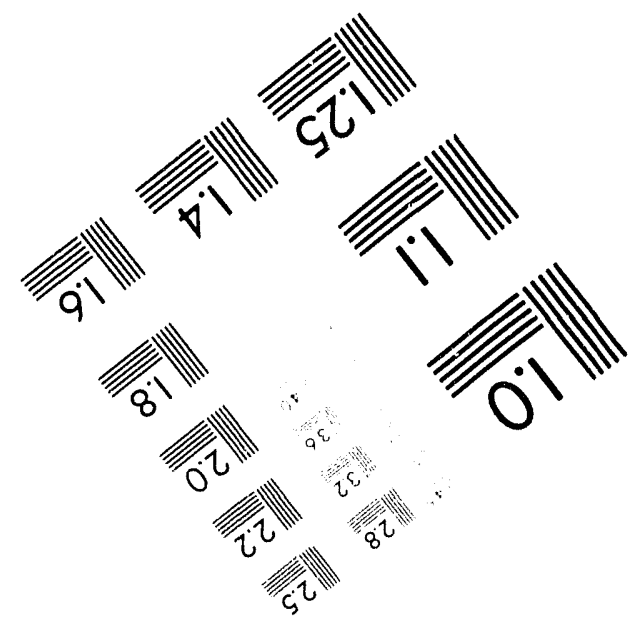



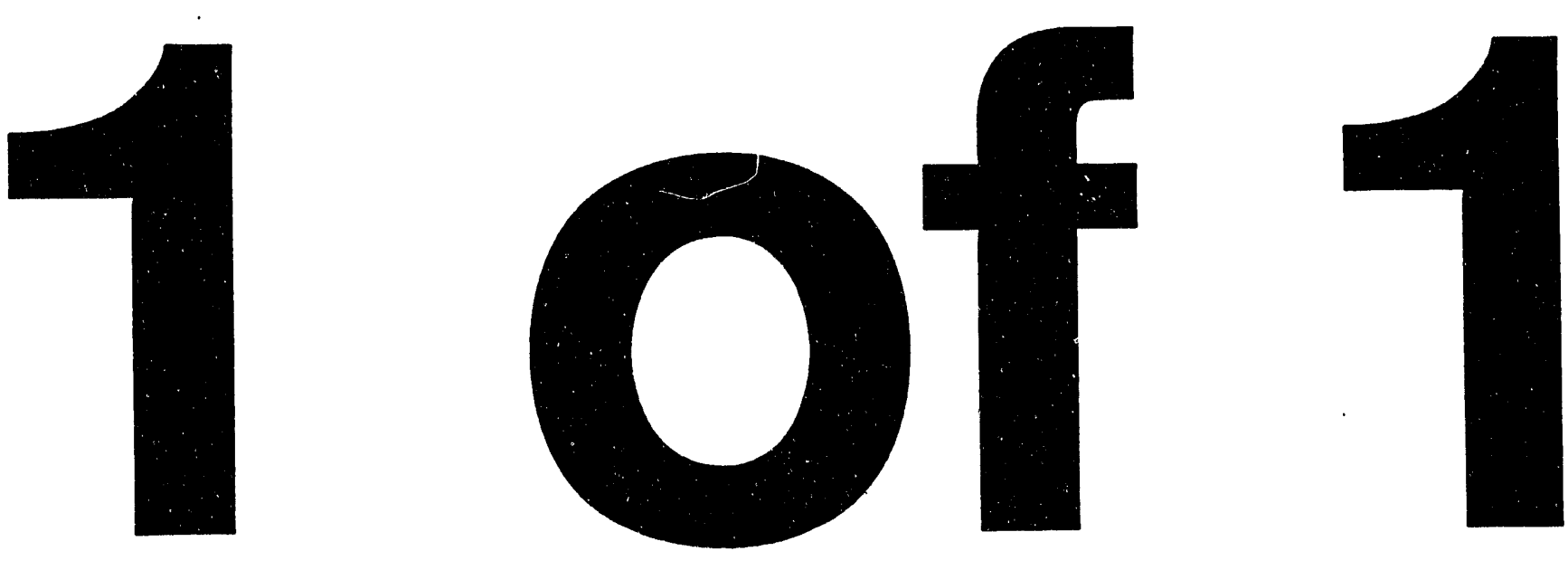


\section{A Two-Level Formal Specification of a Defense Communications System}

by G.H. Chisholm and R.A. Kemmerer*

Decision and Information Sciences Division,

Argonne National Laboratory, 9700 South Cass Avenue, Argonne, Illinois 60439

April 1994

Work sponsored by the U.S. Department of Defense, National Security Agency

"Kemmerer is affiliated with the Reliable Software Group, Department of Computer Science, University of California at Santa Barbara. 


\section{CONTENTS}

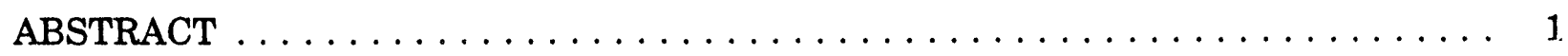

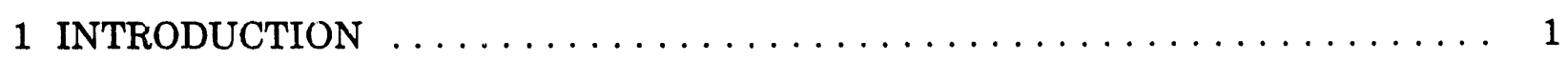

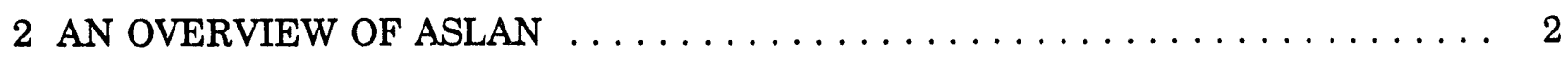

3 FORMAL PRELIMINARY DESIGN SPECIFICATION FOR THE SYSTEM $\ldots \ldots \quad 4$

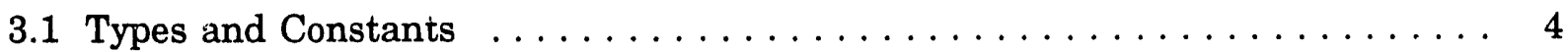

3.2 State Variables ............................... 6

3.3 Critical Requirements - Invariants and Constrainis $\ldots \ldots \ldots \ldots \ldots \ldots .7$

3.4 Initial Conditions $\ldots \ldots \ldots \ldots \ldots \ldots \ldots \ldots \ldots \ldots \ldots \ldots \ldots, 8$

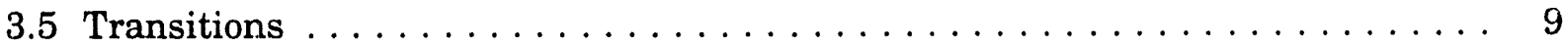

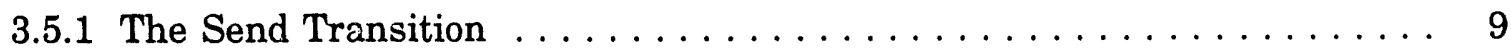

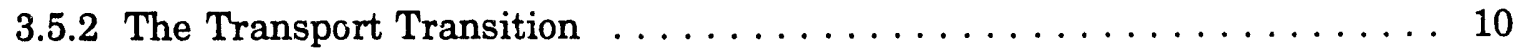

3.5.3 The Receive Transition $\ldots \ldots \ldots \ldots \ldots \ldots \ldots \ldots \ldots \ldots \ldots \ldots$

4 VERIFICATION OF THE PRELIMINARY DESIGN SPECIFICATION . . . . . 12

5 A DETAILED FORMAL SPECIFICATION OF THE SYSTEM $\ldots \ldots \ldots \ldots \ldots 13$

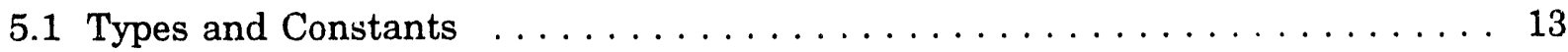

5.2 State Variables . . . . . . . . . . . . . . . . . . . . . . . . . 14

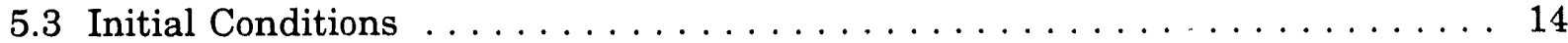

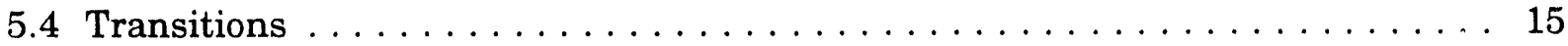

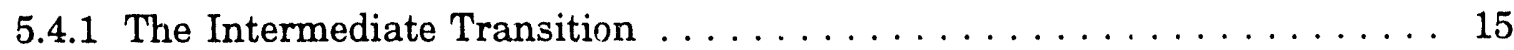

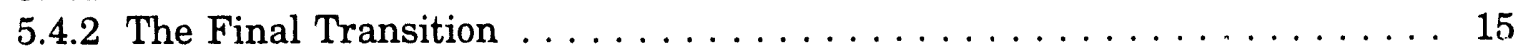

5.4.3 The Remove Links Transition $\ldots \ldots \ldots \ldots \ldots \ldots \ldots \ldots \ldots \ldots$

5.4.4 The Restore Links Transition $\ldots \ldots \ldots \ldots \ldots \ldots \ldots \ldots \ldots \ldots, 16$

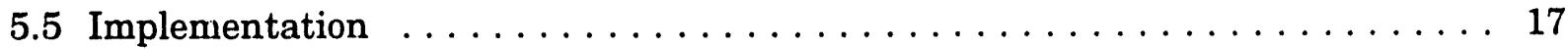

6 VERIFICATION OF THE DETAILED DESIGN SPECIFICATION $\ldots \ldots \ldots \ldots 18$

7 CONCLUSIONS AND FUTURE WORK $\ldots \ldots \ldots \ldots \ldots \ldots \ldots \ldots \ldots \ldots \ldots$

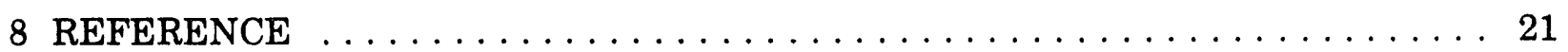

APPENDIX: ASLAN Formal Specification for the System $\ldots \ldots \ldots \ldots \ldots \ldots 23$

\section{FIGURES}

1 The Defense Communications System Specified in the Preliminary Design . . . . . 4

2 The ASLAN System Specified in the Detailed Design $\ldots \ldots \ldots \ldots \ldots \ldots$ 


$$
\text { - }
$$




\title{
A TWO-LEVEL FORMAL SPECIFICATION OF A DEFENSE COMMUNICATIONS SYSTEM
}

by

\author{
G.H. Chisholm and R.A. Kemmerer
}

\begin{abstract}
A two-level ASLAN formal specification of a defense communications system is presented. The ASLAN model is designed to enhance the understanding of critical requirements and demands of the defense communications system. For the top-level (high-level) specifications, the structural details of the actual network are abstracted to allow more time for examining the interactions between the sites and the network. At this level, DataGrams move through the network, although the actual routing decisions are not specified. More details are added in the second-level specification. At this level, structure is added to the network.
\end{abstract}

\section{INTRODUCTION}

Computer systems are being used in critical situations with sensitive data, which makes it very important to ensure that these systems perform as desired. The defense communications system contains particularly sensitive data. The purpose of this report is to demonstrate ways in which formal specification techniques can be used to model the critical requirements for this system, thereby leading to a better understanding of the requirements. The goal is to ensure that the requirements are appropriate and that they are correctly implemented.

Section 2 presents a brief overview of the ASLAN $^{1}$ formal specification language and the ASLAN specification processor. Section 3 discusses the formal preliminary design specifications for the defense communications system. Section 4 gives proof obligations automatically generated by the ASLAN processor for these top-level (hereafter referred to as "high-level") specifications. Section 5 presents the detailed design specification and elaborates on the network structure. Section 6 discusses the proof obligations for this detailed specification. Finally, Section 7 states conclusions and future work plans.

1 The original Aslan was the noble lion in C.S. Lewis's Chronicles of Narnia (Macmillan Publishing Company, New York, N.Y., 1955). Aslan created Narnia and gave the animals the gift of speech. 


\section{AN OVERVIEW OF ASLAN}

The name for the formal specification language and the specification language processor is ASLAN. The ASLAN specification language is an extension of first-order predicate calculus with equality and uses the state machine approach to specification. The system being specified is in various states, and states are differentiated by the values of the state variables. The values of these variables can be changed only by using well-defined state transitions. The key elements of the ASLAN language are types and constants, state variables, invariants and constraints, initial conditions, and transitions.

Predicate calculus assertions specify the desired properties that must hold at every state and between two consecutive states. Critical requirements that must be met in every state are known as state invariants. These invariants specify the critical requirements for a good state (i.e., a secure state), and they must be shown to hold for all reachable states, including the initial state. To prove that a specification satisfies the critical requirements, the ASLAN specification processor automatically generates the proof obligations needed to construct an inductive proof of the correctness of the specification with respect to the invariant assertions.

The critical requirements that must hold between two consecutive states are ASLAN constraints. These constraints detine secure state transitions (i.e., requirements that must be satisfied by every state transition). The constraint is included as part of the proof obligations generated to construct the inductive proof.

An ASLAN specification consists of a sequence of levels. Each level is an abstract data-type view of the system being specified. The high-level view is an abstract model that defines:

- What constitutes the system (i.e., types, constants, and variables);

- What the system does (i.e., state transitions); and

- What critical requirements the system must meet (i.e., invariants and constraints).

Low levels become increasingly detailed, with the lowest level corresponding closely to highlevel code. The ASLAN specification processor generates correctness conjectures whose proofs ensure that low levels correctly refine high levels.

The ASLAN specification processor has been implemented to parse ASLAN statements that specify different levels of abstraction as well as the critical requirements. The processor also produces the proof obligations needed (1) to prove a specification is correct with respect to the critical requirements and (2) to show the correct refinement of levels. The ASLAN language and use of the language processor are detailed in the ASLAN User's Manual (Auernheimer and Kemmerer 1992). 
Before formally specifying the example system, it is useful to have a basic understanding of some of the notation required by ASLAN. The following symbols are used for logical operations:

\& Logical AND

I Logical OR

Logical NOT

$\rightarrow$ Logical implication

In addition, a conditional form is used:

IF A THEN B ELSE C FI,

where $\mathrm{A}$ is a predicate, and $\mathrm{B}$ ard $\mathrm{C}$ are well-formed terms. The notation for set operations is as follows:

$\begin{array}{ll}\text { ISIN } & \text { is a member of } \\ \text { UNION } & \text { set union } \\ \text { CONTAINED_IN } & \text { subset } \\ \text { SET_DIFF } & \text { set difference } \\ \text { \{a,b..c\} } & \text { the set consisting of elements a,b..and c }\end{array}$

The ASLAN language also contains the following quantifier notation:

FORALL

for all

EXISTS there exists

Finally, a special ASLAN symbol (a prime symbol) is used to indicate the old value of a variable (e.g., $x^{\prime}$ is the value of variable $x$ in the previous state). 


\section{FORMAL PRELIMINARY DESIGN SPECIFICATION FOR THE SYSTEM}

In this section, each of the components of the ASLAN preliminary design specification for the defense communications system is discussed in detail. The appendix gives the complete ASLAN specification for the system. Figure 1 represents the system specified in the preliminary design.

At this level of detail, the network is unstructured. Each of the sites interfaces with the network through two buffers: a send buffer and a receive buffer. A site places DataGrams in its send buffer when they are ready to be transmitted to another location. The network transports the DataGrams to the destination, where they are placed in that location's receive buffer. Details of the movement of DataGrams within the network are unknown at this time.

\subsection{TYPES AND CONSTANTS}

ASLAN is a strongly typed language; that is, every simple and constructed type must be declared in the type section of the specification. At this stage in the design of the

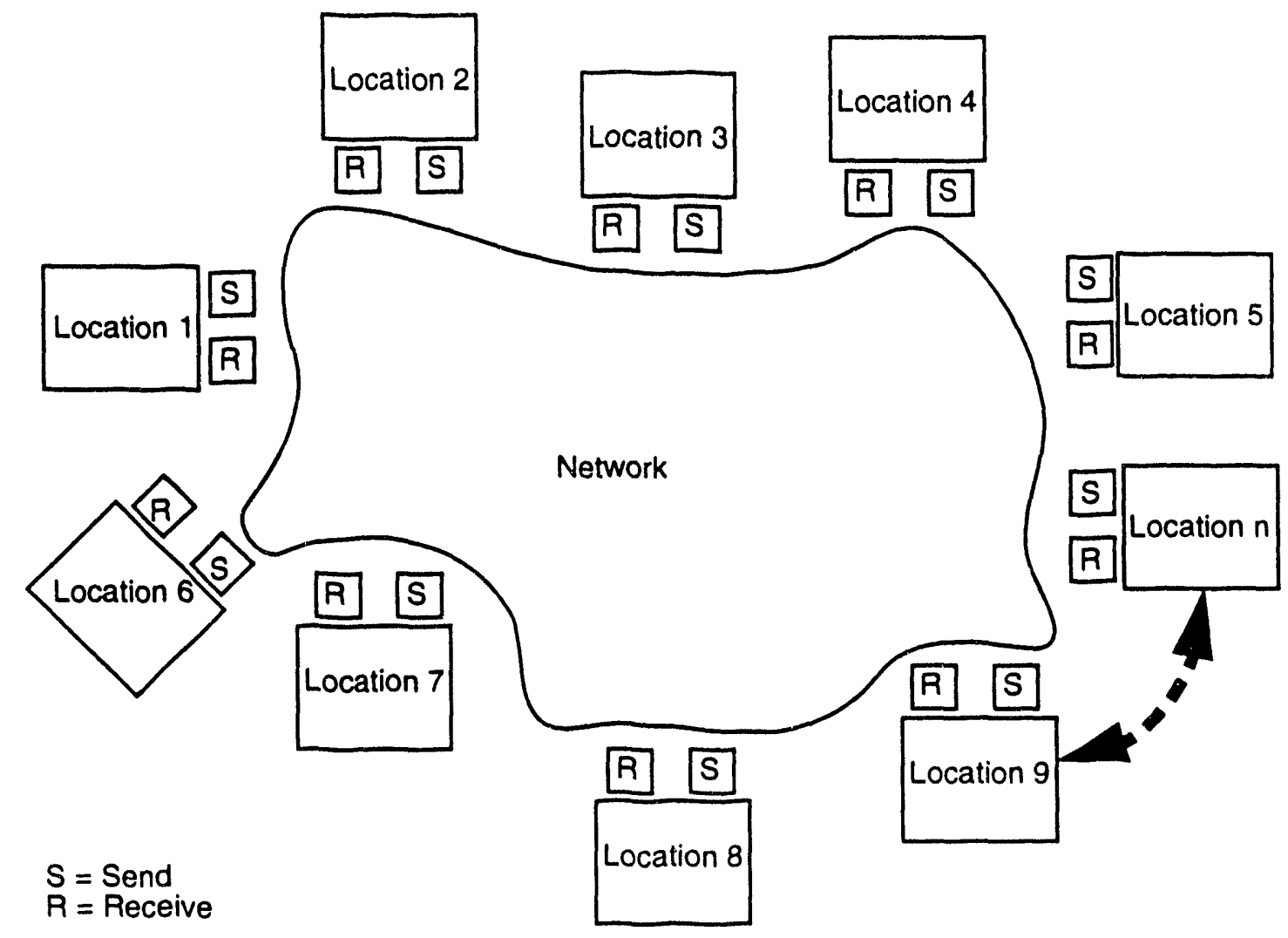

FIGURE 1 The Defense Communications System Specified in the Preliminary Design 
communications system, the structure of the network is unknown; however, it is assumed that to ensure that the design contains the required proporties, the network will contain DataGrams. Thus, the DataGram is the first type declared in the type declaration section of the specification. Again, the specific structure of a DataGram is unknown, as are the possible values that an element of that type can assume.

The declaration

DataGram

indicates an "unspecified type." The only relations available on elements of an unspecified type are "=" and " $\neq . "$

Although its structure is unknown, a DataGram has a source field, a destination field, a data field, and a time stamp. These values are represented in the specification by the following constants:

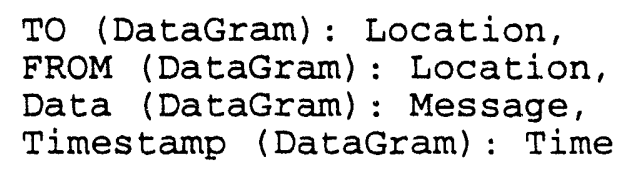

In addition, because DataGrams can be corrupted or phony DataGrams can be injected into the network, it is important to know whether a DataGram is well formed. A well-formed DataGram is indicated by the Boolean constant:

We11_Formed(DataGram): Boolean

The message and location declarations are also unspecified types. It is not important at this level of the design to know the makeup of a location or a message. However, it is necessary to denote that different types of messages and locations occur. For instance, a message can be either a status message or a command message, and a location can be either a battle manager, a sensor, or a weapon. The constants

Type_of_Message (Message): Message_Type,

Type_of_Site (Location): Location_Type

specify the message and location, respectively. The Boolean constant

Consistent_Message (Message_Type,Location_Type) : Boolean

determines whether a message type is consistent with its originating location. The type

Time

is also unspecified because, at this stage in the design process, the units of time have not been specified. 
In ASLAN, the two most commonly used type constructors are sets and lists. In the example specification, DataGrams is a set of DataGram, and Locations is a set of Location:

DataGrams IS SET OF DataGram,
Locations IS SET OF Location

Because some messages in the system are more significant than others, it is important to assign a precedence level with the messages. A declaration of the ASLAN subtype is used for this purpose:

Precedence_Level IS TYPEDEF $T$ : Integer $(T>=1 \& T<=3)$

This subtype declaration specifies that an element of type precedence is a subtype of integer, and its value is in the range from 1 to 3 . The constant

Precedence (Message): Precedence_Level

associates a precedence with a message. The last constant

Neighbor (Location, Location): Boolean

determines whether two locations are one "hop" away from one another.

\subsection{STATE VARIABLES}

As mentioned in Section 2, states are differentiated by the value of the state variables, and state variables are referenced and/or modified by state transitions. All state variables must be declared in the variable section of the formal specification.

The preliminary design specification has five state variables. The first four variables are DataGrams, and the first two of these DataGrams are parameterized by location:

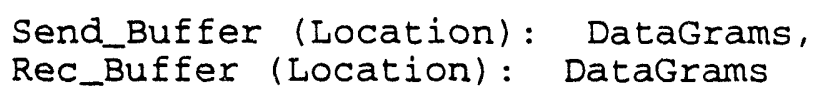

These two variables represent DataGrams that are buffered while they are waiting to be put into the network, as well as DataGrams that have been removed from the network and are waiting to be processed by the site. The third state variable

Network: DataGrams

represents all DataGrams in transit in the network.

The fifth state variable in the specification

Action_Items (Location): DataGrams 
represents DataGrams removed from the receive buffer that await action by the site. Finally, the variable

Now

represents the current time.

\subsection{CRITICAL REQUIREMENTS - INVARLANTS AND CONSTRAINTS}

The defense communications system aims (1) to deliver the most critical DataGrams (i.e., those with the highest precedence) first, (2) to take actions only on well-formed DataGrams, and (3) to allow a DataGram to reside in only one place at a time. These restrictions on moving and processing DataGrams should be specified in the critical requirements.

The ASLAN system expresses the critical requirements as invariants and constraints. The invariants express the critical requirements that must hold in every reachable state, and the constraints express the critical requirements that must hold between two consecutive states. The restrictions that a DataGram can reside in only one place at a time are expressed as invariants in the ASLAN specification. The first conjunct of the invariant

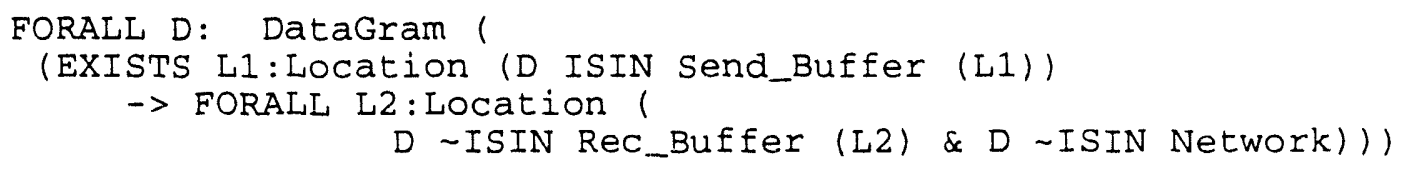

specifies that if a DataGram is in the send buffer of a particular location, it is neither in the network nor in a receive buffer. The fifth conjunct

FORALL L1, L2: Location (

D ISIN Send_Buffer(L1) \& D ISIN Send_Buffer(L2) $\rightarrow$ L1=L2)

further states that the DataGram can be in the send buffer at only one site.

The second and fourth conjuncts of the invari int specify similar properties for the receive buffers. The third conjunct specifies that a Data' 'ram in the network cannot reside in a send buffer or in a receive buffer.

Before examining the constraints, recall that a primed variable (e.g., $x^{\prime}$ ) in an ASLAN expression indicates the value assigned to that variable in the previous state. The example system has four constraints. The first conjunct of the constraint 


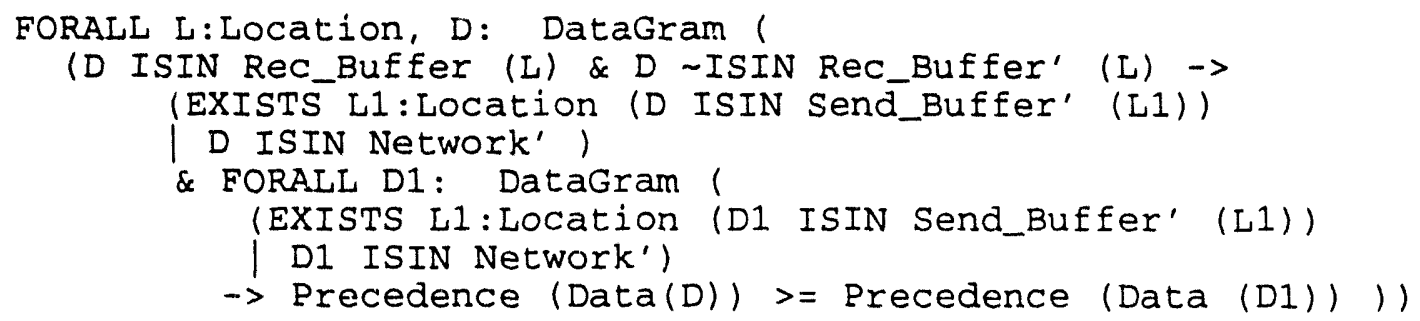

expresses the requirement that if a new DataGram is placed in the receive buffer of a location, it must have previously been in the network or in some site's send buffer. That is, the communications system does not generate DataGrams. Furthermore, this conjunct specifies that other DataGrams of higher precedence cannot reside in the network or in a site's send buffer. The second conjunct specifies a similar requirement for DataGrams moving from a send buffer to either the network or a receive buffer.

The third conjunct

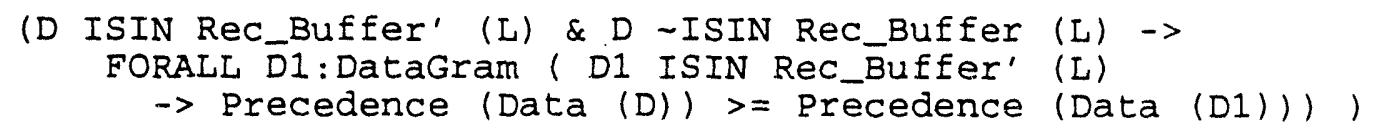

is a local restriction on each site. It requires each location to process high-priority messages first.

The last conjunct of the constraint

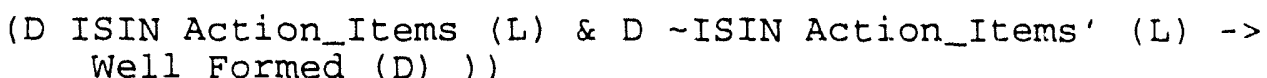

is also local. It specifies that only well-formed DataGrams should become action items.

\subsection{INITIAL CONDITIONS}

The initial conditions of an ASLAN formal specification express the restrictions on the initial state of the system. That is, for each state variable, it is necessary to express the restrictions to be placed on its initial value. Because the initial state is a reachable state, it is necessary for the initial state to satisfy the invariant. (This condition is one of the proof obligations automatically generated by the ASLAN specification processor.) Therefore, the invariant could be used as the initial expression. A more realistic approach is to choose a degenerate case that reflects the initial introduction of the system being specified into the operational environment while still satisfying the invariant. This approach is used in the specifications for the defense communications system. 
The initial condition specifies that the send and receive buffers for all sites contain no DataGrams. In addition, the network contains no DataGrams. This fact is expressed by

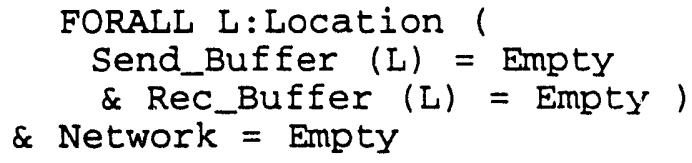

\subsection{TRANSITIONS}

ASLAN transitions specify the ways in which a system can change from one state to another. A transition is composed of a header, an entry assertion, and an exit assertion. The header gives type information for its parameters. The entry assertion expresses the conditions required to have the transition. The exit assertion specifies the resultant state after the transition occurs; that is, it specifies values of the state variables in the new state relative to values in the previous state.

The preliminary design specification for the defense communications system contains three transitions: Send, Transport, and Receive. These transitions correspond to putting DataGrams in the send buffer for transmission; transporting them through the network to their destination, where they are placed in the receive buffer; and processing them in the receive buffer and taking action, if necessary.

\subsubsection{The Send Transition}

The parameters of the Send transition are the sender, a list of receivers, and the message to be sent. The Send transition represents what occurs when a message is placed in possibly multiple DataGrams with multiple destinations.

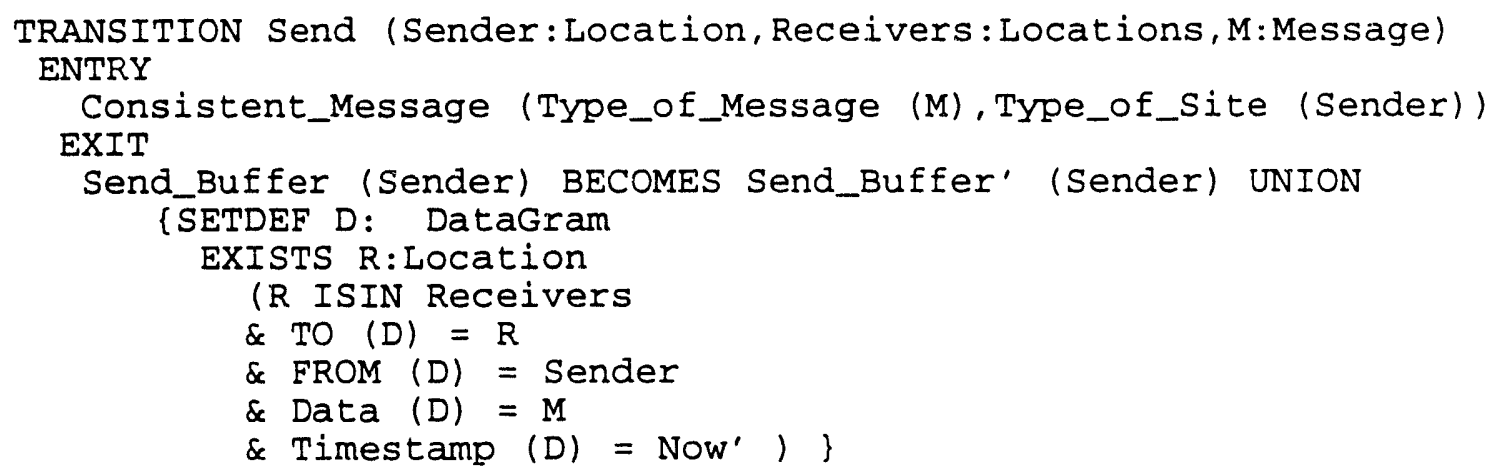

The entry assertion for this transition specifies that the message sent must be consistent with the sending site. For example, a sensor site could not send a launch command.

The exit assertion specifies that a DataGram for each location in the receiver set is placed in the sender's send buffer. The content of each DataGram contains the destination 
for its TO field, the sender's location for its FROM field, the message for its data field, and the current time (the value of state variable Now) for a time stamp. The BECOMES operator used in this expression is shorthand notation provided by ASLAN. This notation asserts that the value of a parameterized variable changes for some arguments but remains unchanged for the other arguments. Thus, the exit assertion is equivalent to

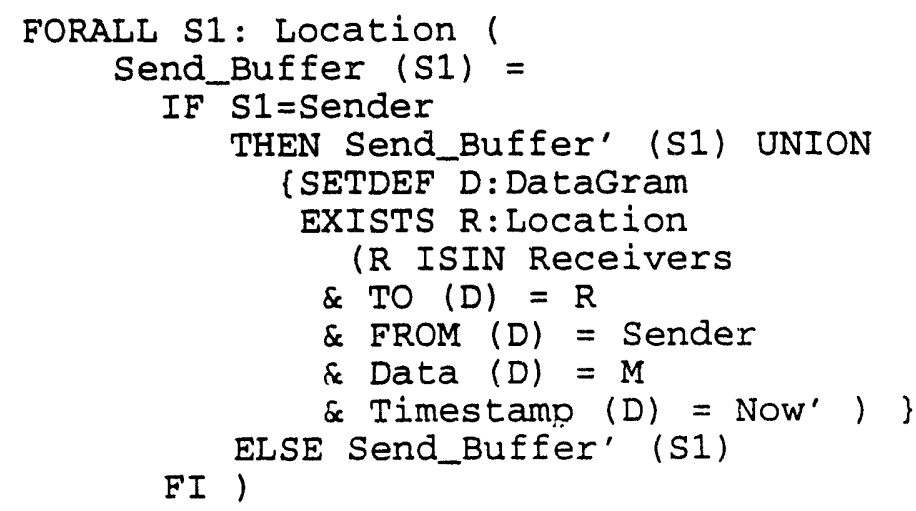

The ASLAN specification processor transforms BECOMES statements to this form when constructing the proof obligations.

\subsubsection{The Transport Transition}

The Transport transition models the movement of DataGrams in the communications system. Each time this transition is fired, a single DataGram moves from a location's send buffer or from part of the network to either the receive buffer at the destination site or to another part of the network.

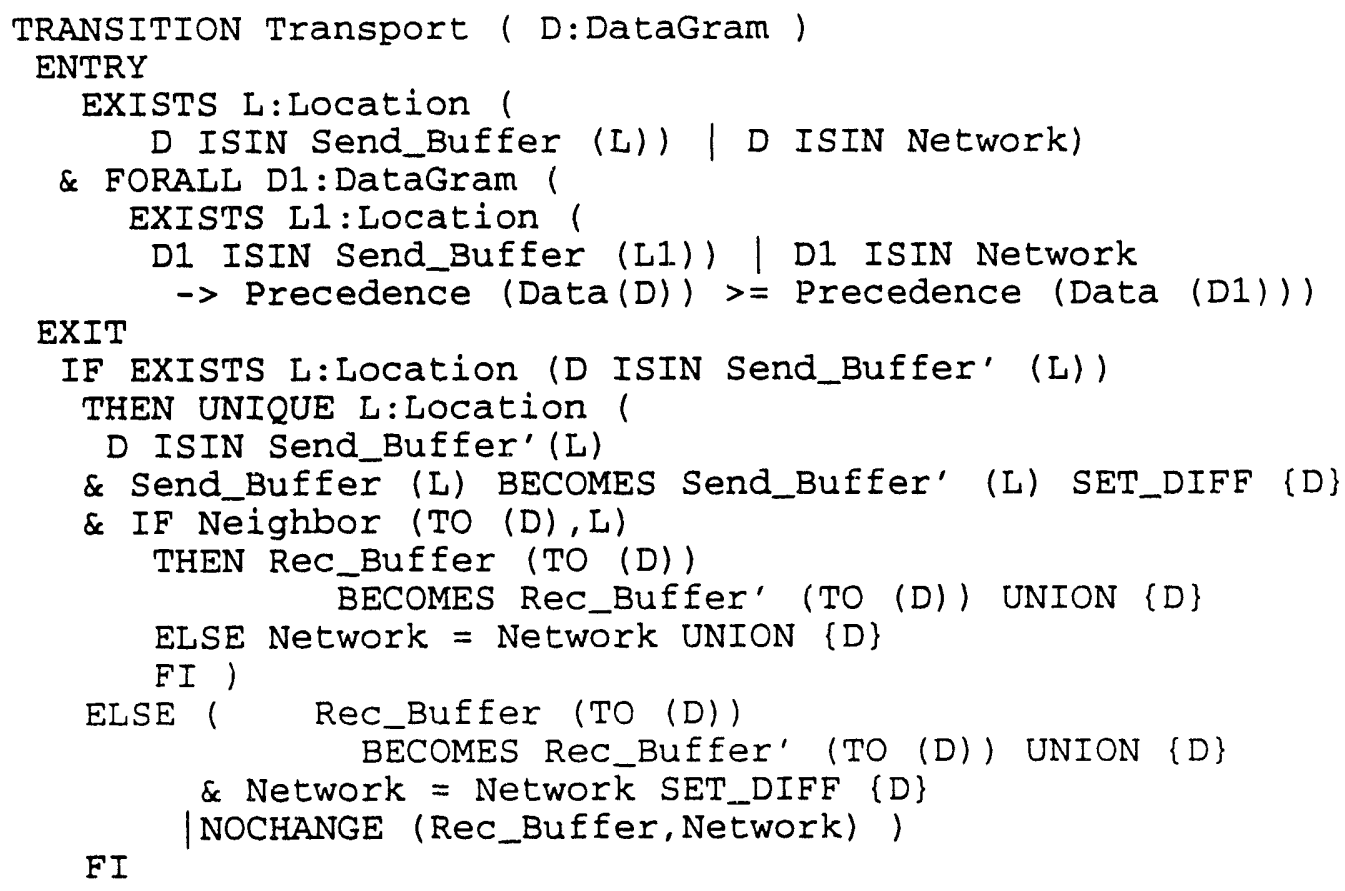


The first conjunct of the entry assertion for the Transport transition specifies that the DataGram must be in either a site's send buffer or the network. The second conjunct specifies that the precedence of this DataGram is at least as high as any DataGram located in a send buffer or the network.

The exit assertion is written by using the ASLAN conditional form (IF THEN ELSE FI). The THEN form corresponds to what occurs if the DataGram is in a send buffer; the ELSE form corresponds to what occurs if the DataGram is in the network. Suppose the DataGram is in a send buffer and is being sent to a neighboring site of the site that is sending the message. In that case, the DataGram is placed in the receive buffer at the destination site; otherwise, it is put in the network. In both cases, the DataGram is removed from the send buffer. When a DataGram is already in the network, it is either placed in the receive buffer at the destination site and removed from the network or allowed to remain in the network. The latter option is a disjunction because at this level of detail it is not known how it is decided whether the DataGram remains in or is removed from the network. This process will be clarified in the more detailed second-level specification.

\subsubsection{The Receive Transition}

The Receive transition represents a site that processes DataGrams that are delivered to its receive buffer.

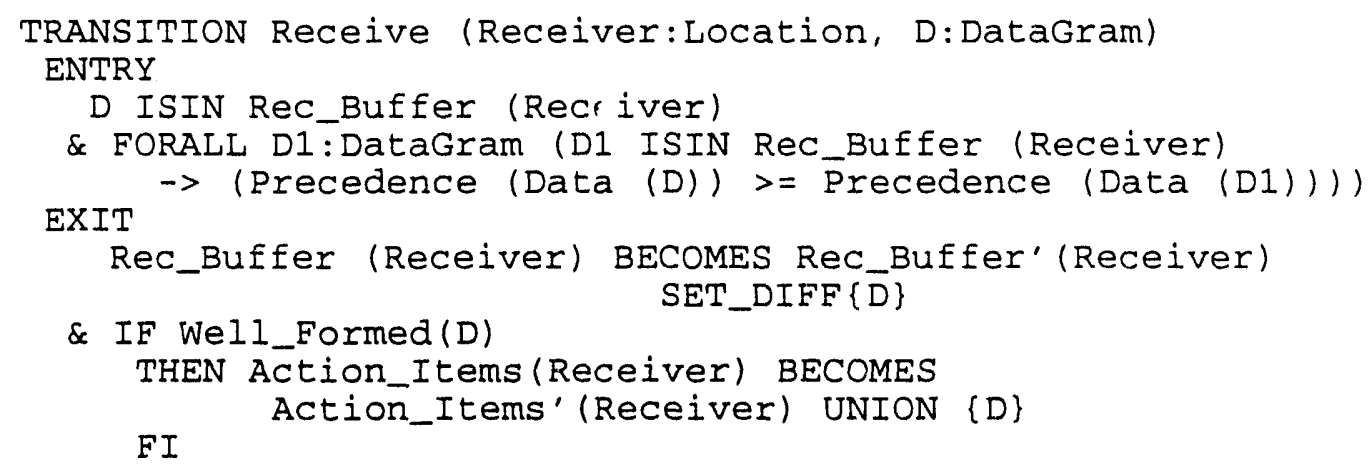

The entry assertion specifies that only DataGrams in the site's receive buffer can be processed and that the precedence of the DataGram processed is at least as high as other DataGrams in this site's receive buffer.

The exit assertion specifies that the DataGram is removed from the site's receive buffer and that other DataGrams remain in the buffer. It also specifies that a well-formed DataGram is added to the list of action items for this site. At this level, the well-formed property is not defined; however, cryptographic check sums could be used to implement the well-formed check. 


\section{VERIFICATION OF THE PRELIMINARY DESIGN SPECIFICATION}

Upon completion of the preliminary design specification for the defense communications system, the specification was input into the ASLAN processor to produce the necessary proof obligations. The aim was to guarantee that the transitions specified would satisfy the formal critical requirements. Before examining the proof obligations generated, recall that the formal critical requirements consist of invariants and constraints, where an invariant is a relationship between state variables that must hold in every reachable state, and a constraint is a relationship between state variables that must hold between two consecutive states.

The ASLAN proof methodology uses an inductive approach to generate the necessary proof obligations to ensure that the critical requirements are preserved. In this approach, the invariants are proved for the initial state. For every transition, it is then necessary to show that if the transition fires in a state where the invariants hold, the resultant state also satisfies the invariants. In addition, the previous and new states must satisfy the relationships expressed by the constraints; that is, the initial state is the base case, and the induction is on the transitions. Thus, the transitions can be fired in any order, and, by induction, any reachable state will satisfy the invariants, and any two consecutive states will satisfy the constraints.

The first proof obligation generated by the ASLAN processor is known as the Initial Conditions Theorem:

$$
I N I T \rightarrow I N V,
$$

where $I N I T$ is the initial clause, and INV represents the invariants in the invariant clause of the specification.

In addition, for each transition in the specification, the ASLAN processor generates a Transition Theorem:

$$
I N V^{\prime} \& E N T R Y^{\prime} \& E X I T \rightarrow I N V \& C O N
$$

where ENTRY and EXIT are the entry and exit assertions for the transition, and CON is the constraint clause. (It is beyond the scope of this report to discuss the proofs of these correctness conjectures.) 


\section{A DETAILED FORMAL SPECIFICATION OF THE SYSTEM}

Once additional design decisions have been made, a more detailed formal design specification can be developed. The detailed design specification will give more information about the structure of the network. Figure 2 shows the system specified in the detailed design. At this level, the network is an architecture of static links, with each link connecting two sites. These links can be disabled and restored. In addition, each location has a transfer buffer to hold DataGrams in transit from their source to their destination.

Many of the types, constants, and variables of the preliminary design specification are identical in the detailed design specification. That is, no new details have been added for these entities. The following sections discuss only the new or modified entitls of the specification.

\subsection{TYPES AND CONSTANTS}

Two new types have been added to the detailed design:

Link,

Links IS SET OF Link

A link connects two sites and can become inoperable and then operable again (Section 5.4).

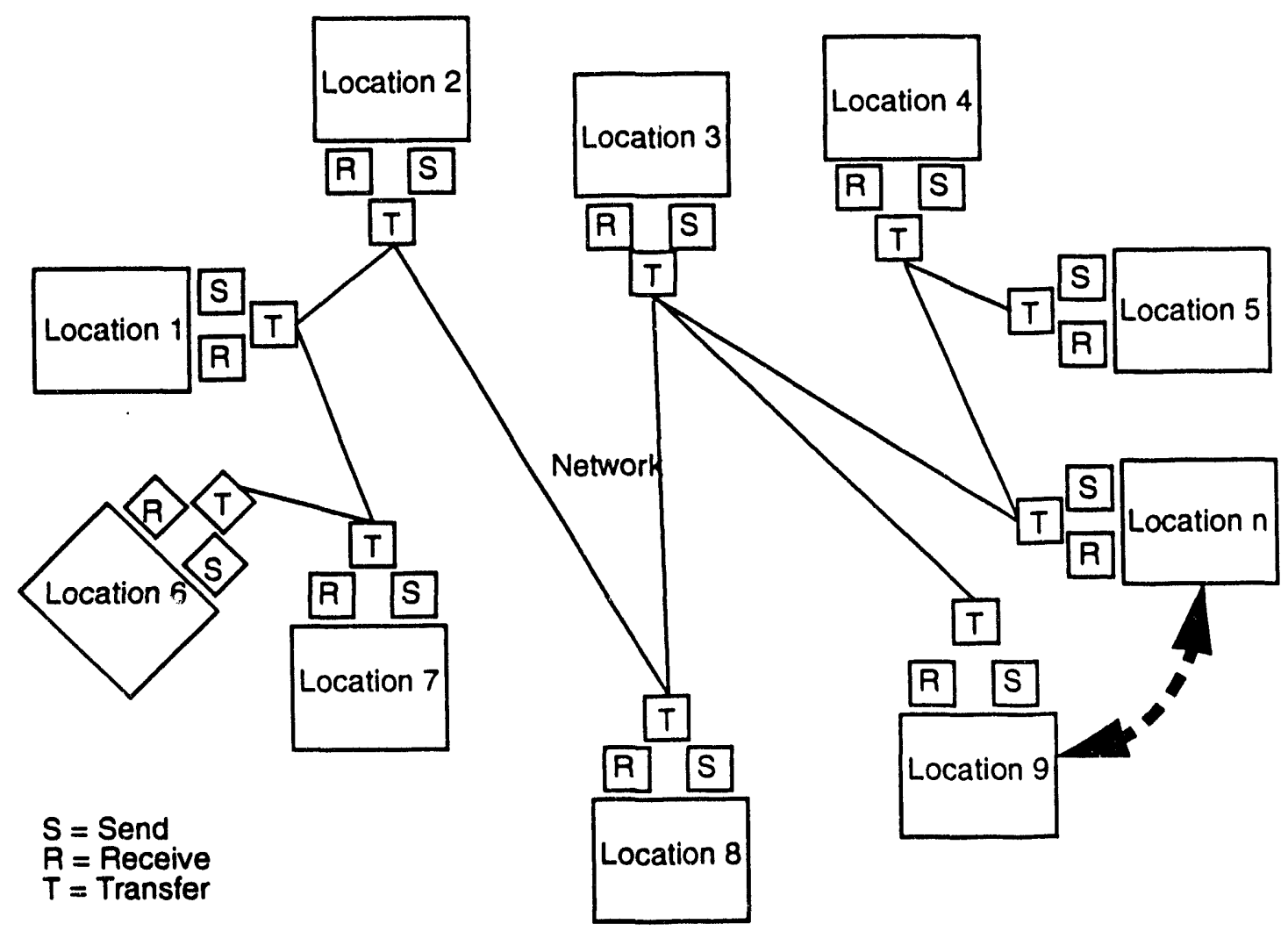

FIGURE 2 The ASLAN System Specified in the Detailed Design 
The constant

Next_Hop (Location, Location):Location

represents the routing algorithm used. At this level of detail, no actual algorithm is present; however, given any two locations, the algorithm determines the next location along the path.

The constant

Net_Arch:Links

is a set of links that indicates pairs of sites that are connected. Note that linkage between two sites does not mean that the link can be used for communication. That is, although the architecture is static, the operability of the links is dynamic. Therefore, some links may be inoperable and unavailable for communication.

Another new constant declared

Link_ok (Location, Location, Links) :Boolean

determines whether a link between two locations exists. That is, given two locations and a set of operable links, this constant is true if the link between the two locations is in the set; otherwise, it is false.

\subsection{STATE VARIABLES}

As mentioned above, a transfer buffer is associated with each site. The state variable Trans_Buffer (Location) : DataGrams

represents these buffers.

A second new state variable introduced in the detailed design specification is Operational_Links:Links

This state variable represents those links in the network that are currently operational.

\subsection{INITIAL CONDITIONS}

The initial conditions clause specifies that neither the send nor the receive buffers contain DataGrams. In addition, all transfer buffers are empty. The initial conditions clause also specifies that all links in the network are currently operational. This condition is expressed by

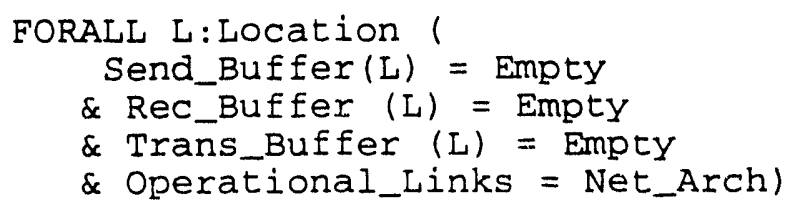




\subsection{TRANSITIONS}

Six transitions are included in the detailed design specification for the defense communications system: Send, Intermediate, Final, Receive, Remove Links, and Restore Links. The Send and Receive transitions are identical to those specified in the preliminary design. The Intermediate and Final transitions are realizations of the Transport transition in the preliminary design, and the two remaining transitions do not correspond to transitions specified in the preliminary design. Each of the new transitions is discussed in Sections 5.4.1-5.4.4, respectively.

\subsubsection{The Intermediate Transition}

The Intermediate transition implements the Transport transition that is fired when the DataGram is being transferred to a destination other than its final destination.

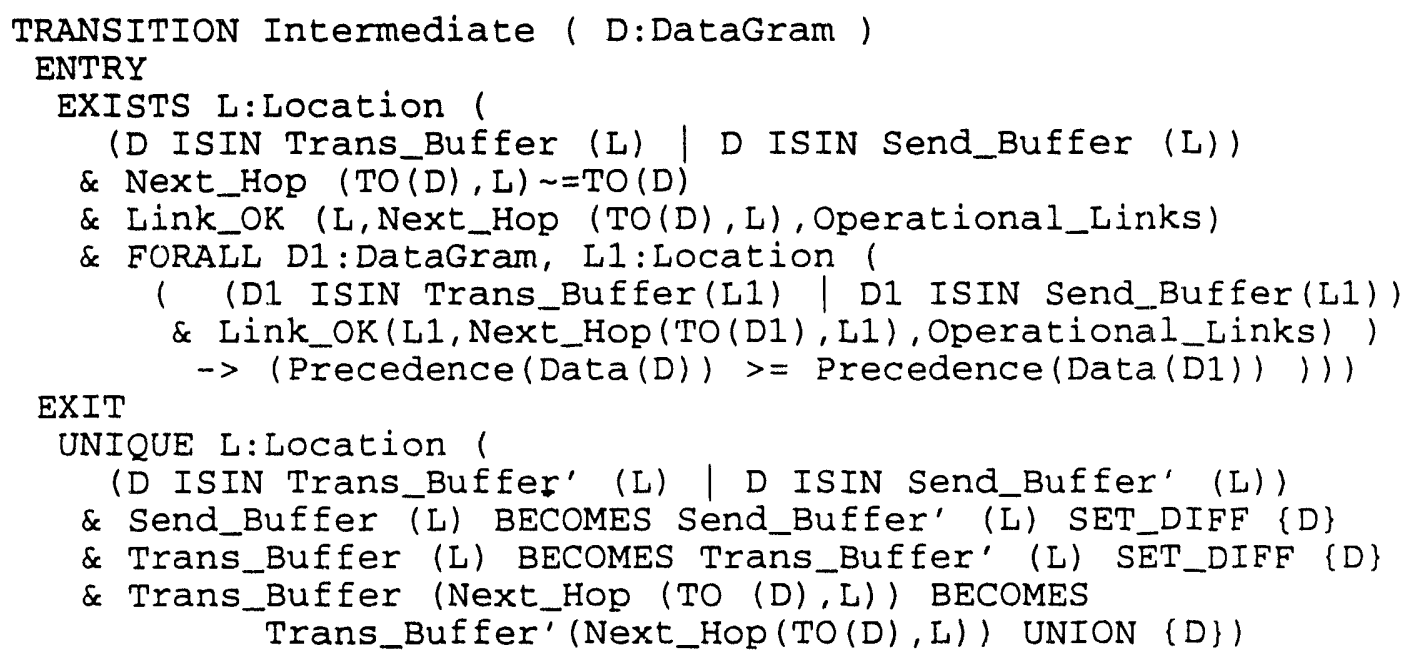

The first conjunct of the entry assertion for this transition specifies that the DataGram must be in either a site's send buffer or a transfer buffer. The second conjunct specif, 3 that the next location is not the destination, and the third conjunct specifies that an operational link exists between the DataGram's present location and its next location. The final conjunct specifies that the precedence of this DataGram is at least as high as any DataGram located in a send buffer or a transfer buffer; it also identifies which link becomes operational next.

The exit assertion for this transition specifies removal of the DataGram from either the send buffer or the transfer buffer where it currently resides. The DataGram is placed in the transfer buffer of the site that is next in its routing (as determined by the next hop constant).

\subsubsection{The Final Transition}

The Final transition implements the Transport transition that is fired when the next location for the DataGram being transferred is its destination location. 


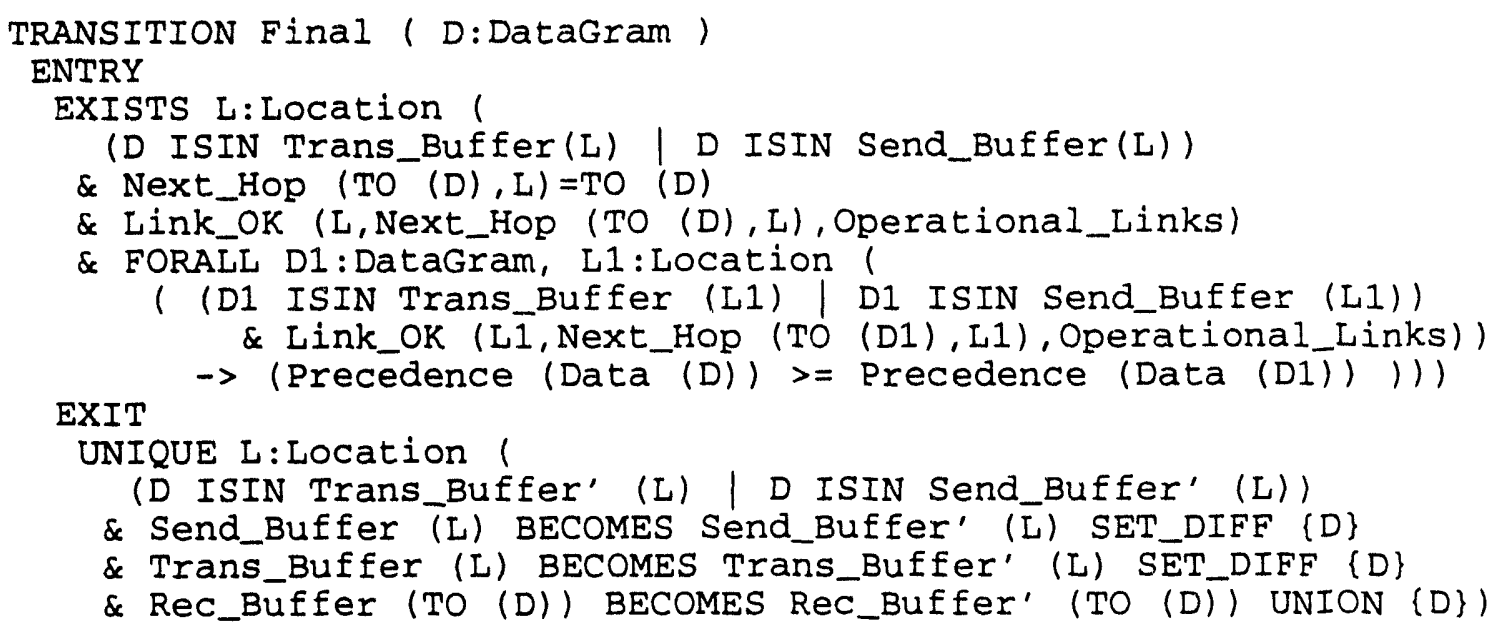

The entry assertion for the Final transition differs from the entry assertion for the Intermediate transition only in the second conjunct. This conjunct specifies that the next hop is the DataGram's destination. The exit assertion for the Final transition differs from the exit assertion for the Intermediate transition in one way; that is, the DataGram is placed in the receive buffer of the site that corresponds to its next hop instead of in the site's transfer buffer.

\subsubsection{The Remove Links Transition}

The Remove Links transition models a set of links of the network that becomes inoperable. For example, an electromagnetic pulse could cause some of the links to fail.

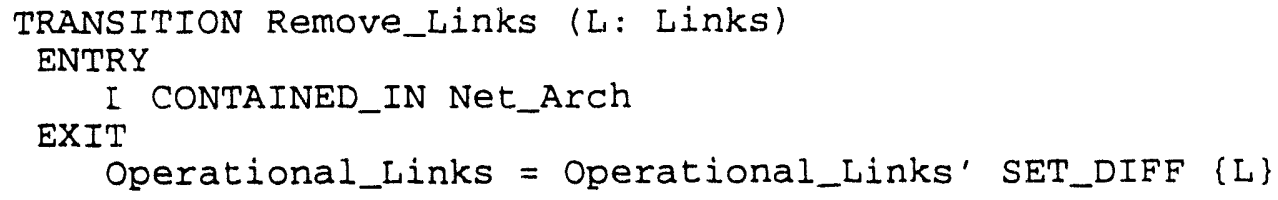

One entry condition exists for the Remove Links transition: links that are parameters to the transition must be links in the network architecture. The exit assertion specifies that these links are no longer operable; therefore, they are removed from the set of operational links.

\subsubsection{The Restore Links Transition}

The Restore Links transition models a set of links being restored to operational status.

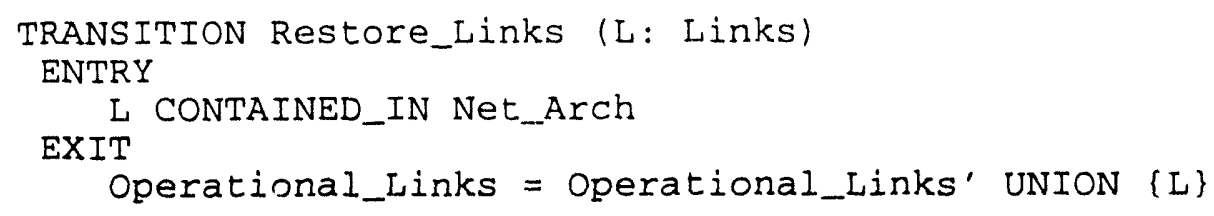

The entry assertion for this transition requires the links to be links in the network architecture. The exit assertion adds these links to the set of operational links. 


\subsection{IMPLEMENTATION}

The implementation section indicates how each of the types, constants, variables, and transitions that occur at a high level is refined in the level immediately below (i.e., the level containing the implementation section). For the defense communications specification, most mappings are identity maps, except for the constant neighbor, the variable network, and the Transport transition.

The mapping for the constant neighbor is

Neighbor $(L 1, L 2)==$ Next_Hop $(L 1, L 2)=L 2$

This mapping specifies what was termed a "neighbor" in the preliminary design specification is now a site that is exactly one hop away.

The mapping for the variable network is

$$
\begin{aligned}
& \text { Network }==\text { (SETDEF D:DataGram EXISTS L:Location ( } \\
& \text { DISIN Trans_Buffer (L) ) \} }
\end{aligned}
$$

The network, which was a collection of DataGrams in the preliminary design specification, is realized by the collection of DataGrams that resides in the transfer buffers. Note that links that are new at this level of detail do not appear in the mapping because they do not correspond to high-level links.

The mapping for the Transport transition is

$$
\text { Transport (D) == Final (D) | Intermediate (D) }
$$

This mapping specifies that the Transport transition can be fired through either the Intermediate or the Final transition. The mapping does not indicate under what conditions one or the other of these transitions fires. These conditions are determined by the entry assertions of the two low-level transitions (Section 5.4). 


\section{VERIFICATION OF THE DETAILED DESIGN SPECIFICATION}

The ASLAN specification processor also generates the appropriate proof obligations for the detailed design specification of the defense communications system. These proof obligations guarantee that the detailed design specification correctly refines the preliminary design specification.

When using ASLAN, the proof obligations between any two levels of specification are always of the same form. First, a proof obligation is generated that guarantees that the initial condition specified in the detailed design (low-level) specification implies the initial condition of the "parent" specification. In addition, for each transition in the preliminary design (high-level) specification, proof obligations are generated to guarantee that the transition is correctly refined in the "child" specification. Finally, for each low-level transition that does not refine a high-level transition, a proof obligation is generated to guarantee that the transition preserves a mapping of the invariant and the constraint.

The low-level specification contains more details (i.e., it is less abstract) than those contained in the high-level specification. Therefore, it is necessary to use the implementation section of the low-level specification to rigorously transform the high-level types, constants, and variables to the entities of the low-level specification.

The interlevel proof obligations generated by the ASLAN specification processor are an Initial Condition Mapping Theorem and Transition Mapping theorems.

The Initial Condition Mapping Theorem is

$$
I N I T_{\text {low }} \rightarrow \operatorname{Impl}\left(I N I T_{\text {high }}\right),
$$

where $I N I T_{\text {low }}$ is the initial clause of the low-level specification, and $I N I T_{\text {high }}$ is the initial clause of the high-level specification. The $I m p l$ function in this formula denotes the application of the mappings defined in the low-level specification to the types, constants, and variables that appear in the expression to which it is applied.

The Transition Mapping theorems for a high-level transition refined by a single lowlevel transition are

$$
\operatorname{Impl}\left(E N T R Y_{\text {high }}\right) \& \operatorname{Impl}\left(I N V_{\text {high }}\right) \rightarrow E N T R Y_{\text {low }}
$$

and

$$
\operatorname{Impl}\left(E N T R Y^{\prime}{ }_{h i g h}\right) \& \operatorname{Impl}\left(I N V^{\prime}{ }_{\text {high }}\right) \& E X I T_{l o w} \rightarrow \operatorname{Impl}\left(E X I T_{\text {high }}\right),
$$

where ENTRY low and EXIT low are the entry and exit of the low-level transition, and $E N T R Y_{h i g h}$ and $E X I T_{h i g h}$ are the entry and exit of the parent transition, respectively. 
If the parent transition is mapped to several transitions, this mapping is reflected in the mapping theorem generated. For example, in the defense communications system detailed design specification, the disjunctive mapping for the Transport transition generates the following proof obligations:

$$
\text { Impl }\left(E N T R Y_{\text {transport }}\right) \& \operatorname{Impl}\left(I N V_{\text {high }}\right) \rightarrow\left(E N T R Y_{\text {intermediate }} \mid E N T R Y_{\text {final }}\right),
$$

and

$$
\begin{aligned}
& \text { Impl }\left(E N T R Y_{\text {transport }}\right) \& \operatorname{lmpl}\left(I N V_{\text {high }}^{\prime}\right) \&\left(E X I T_{\text {intermediate }} \mid E X I T_{\text {final }}\right) \rightarrow \\
& \quad I m p l\left(E X I T_{\text {Transport }}\right) .
\end{aligned}
$$

For a transition that does not refine a high-level transition, the proof obligation must guarantee that the invariant and constraint are preserved. The proof obligation generated is

$$
E N T R Y_{\text {low }} \& \operatorname{Impl}\left(I N V^{\prime}{ }_{\text {high }}\right) \& E X I T_{\text {low }} \rightarrow \operatorname{Impl}\left(I N V_{\text {high }}\right) \& \operatorname{Impl}\left(C O N_{\text {high }}\right)
$$

By showing that the low-level initial conditions correctly implement the high-level initial conditions and that the low-level transitions correctly implement the high-level transitions, it follows that the low-level specification preserves the invariants and constraints. That is, because the low-level specification is consistent with the level above it, and the level above is consistent with the formal critical requirements, the low-level specification is consistent with the formal critical requirements. For the defense communications system, the preliminary design specification and the detailed design specification were input to the ASLAN specification processor to generate the necessary proof obligations to ensure that the detailed design specification was consistent with the preliminary design specification. This report has simplified the proof obligations generated by the ASLAN specification processor to clarify the presentation. The details of the actual proof obligations generated are discussed in Auernheimer and Kemmerer (1992). 


\section{CONCLUSIONS AND FUTURE WORK}

The ASLAN formal preliminary design specification and detailed design specification for a defense communications system have been presented. The detailed design specification elaborated on the structure of the network, the design of the routing algorithm, and the possibility of inoperable communication links.

The primary benefit of formally specifying and verifying software is development of more reliable systems. That is, programs developed through the use of this approach perform the desired functions with fewer errors and can be trusted to operate correctly in critical environments. In addition, because undetected errors are usually more expensive to fix once the software becomes operational (as compared with those revealed during the design phase), the cost of software development is reduced. Errors are located and eliminated early in the development process, thus reducing costs.

Formal methods should be integrated into the software development process to ensure that critical systems perform as desired. Using formal notation for the design documents allows rigorous investigation of the design before the code is written. In addition, because critical system requirements are captured in a mathematical notation, the system specification can serve as an unambiguous arbitrator of the desired properties of the system. Furthermore, design properties can be proved, which ensures early in the development process that the system will satisfy its critical requirements. 


\section{REFERENCE}

Auernheimer, B., and R.A. Kemmerer, 1992, ASLAN User's Manual, TRCS84-10, Department of Computer Science, University of California at Santa Barbara, April. 
APPENDIX:

ASLAN FORMAL SPECIFICATION FOR THE SYSTEM 


\section{SPECIFICATION COMMUNICATIONS \\ LEVEL Top_Level}

TYPE

DataGram,

Message,

Location,

Message_Type,

Location_Type,

Time,

DataGrams IS SET OF DataGram,

Locations IS SET OF Location,

Precedence_Level IS TYPEDEF T:Integer(T $>=1 \& \mathrm{~T}<=3$ )

\section{CONSTANT}

TO(DataGram): Location,

FROM(DataGram): Location,

Data(DataGram): Message,

Timestamp(DataGram): Time,

Precedence(Message): Precedence_Level,

Type_of_Message(Message): Message_Type,

Type_of_Site(Location): Location_Type,

Consistent_Message(Message_Type,Location_Type): Boolean,

Well Formed(DataGram): Boolean,

Neighbor(Location,Location): Boolean

\section{VARIABLE}

Send_Buffer(Location):DataGrams,

Rec_Buffer(Location):DataGrams,

Network:Datagrams,

Action_Items(Location):DataGrams,

Now:Time

INITIAL

FORALL L:Location (Send_Buffer $(L)=$ Empty \& Rec_Buffer $(L)=$ Empty) \& Network $=$ Empty

INVARIANT

FORALL D:Datagram (

(EXISTS L1:Location(D ISIN Send_Buffer(L1))

$\rightarrow$ FORALL L2:Location (

D - ISIN Rec Buffer(L2) \& D ISIN Network))

\& (EXISTS L1:Location(D ISIN Rec_Buffer(L1))

$\rightarrow$ FORALL L2:Location (

D ISIN Send_Buffer(L2) \& D ISIN Network))

$\&$ (D ISIN Network

$\rightarrow$ FORALL L:Location (

D - ISIN Rec_Buffer(L) \& D - ISIN Send_Buffer(L)))

\& FORALL L1,L2:Location (

D ISIN Rec_Buffer(L1) \& D ISIN Rec_Buffer(L2) $\rightarrow$ L1 $=$ L2 )

\& FORALL L1,Li2:Location (

D ISIN Send_Buffer(L1) \& D ISIN Send_Buffer(L2) $\rightarrow$ LI=L2 ) ) 
CONSTRAINT

FORALL L:Location, D: DataGram (

(D ISIN Rec Buffer(L) \& D -ISIN Rec_Buffer'(L) $\rightarrow$ ( EXISTS L1:location (D ISIN Send_Buffer'(L1))

ID ISIN Network')

\& FORALL D1:DataGram (

( EXISTS L1:Location(D1 ISIN Send_Buffer'(L1))

( D1 ISIN Network')

$\rightarrow$ Precedence $(\operatorname{Data}(\mathrm{D}))>=$ Precedence $(\operatorname{Data}(\mathrm{D} 1))))$

\&

(D ISIN Send Buffer'(L) \& D - ISIN Send Buffer(L) $\rightarrow$

( EXISTS L1:location (D ISIN Rec_Buffer(L1))

ID ISIN Network)

\& FORALL D1:DataGram (

( EXISTS L1:Location(D1 ISIN Send Buffer'(L1))

( DI ISIN Network')

$\rightarrow>$ Precedence $(\operatorname{Data}(\mathrm{D}))>=$ Precedence $(\operatorname{Data}(\mathrm{D} 1)))$ )

\&

(D ISIN Rec_Buffer'(L) \& D -ISIN Rec_Buffer(L) ->

FORALL DI:DataGram ( DI ISIN Rec_Buffer'(L)

$\rightarrow$ Precedence $(\operatorname{Data}(D))>=$ Precedence $(\operatorname{Data}(D 1)))$ )

$\&$

(D ISIN Action_Items(L) \& D “ISIN Action_Items'(L) $\rightarrow>$ Well_Formed(D) ))

TRANSITION Send ( Sender:Location, Receivers:Locations, M:Message ) ENTRY EXIT

Consistent_Message(Type_of_Message(M), Type_of_Site(Sender))

Send_Buffer(Sender) BECOMES Send_Buffer'(Sender) UNION

[SETDEF D:DataGram

EXISTS R:Location (

R ISIN Receivers

$\& T O(D)=R$

\& $F R O M(D)=$ Sender

$\& \operatorname{Data}(D)=M$

\& Timestamp $(D)=$ Now $)\}$

TRANSITION Transport ( D:DataGram )

ENTRY

EXISTS L:Location (D ISIN Send_Buffer(L)) I D ISIN Network

\& FORALL D1:DataGram (

EXISTS L1:Location (DI ISIN Send Buffer(L1)) | DI ISIN Network

EXIT

$\rightarrow$ Precedence $(\operatorname{Data}(\mathrm{D}))>=$ Precedence $(\operatorname{Data}(\mathrm{Dl})))$

IF EXISTS L:Location (D ISIN Send_Buffer'(L))

THEN UNIQUE L:Location (

D ISIN Send_Buffer'(L)

$\&$ Send_Buffer(L) BECOMES Send_Buffer'(L) SET_DIFF $\{D\}$

\& IF Neighbor(TO(D),L)

THEN Rec_Buffer(TO(D)) BECOMES Rec_Buffer'(TO(D)) UNION \{D\}

ELSE Network $=$ Network UNION $\{$ D $\}$

FI ) 
ELSE ( Rec_Buffer(TO(D)) BECOMES Rec_Buffer'(TO(D)) UNION \{D\} $\&$ Network $=$ Network SET_DIFF $\{$ D $\}$

FI (NOCHANGE(Rec_Buffer,Network))

TRANSITION Receive (Receiver:Location, D:DataGram) ENTRY

D ISIN Rec_Buffer(Receiver)

\& FORALL $\bar{D} 1$ :DataGram (D1 ISIN Rec_Buffer(Receiver)

EXIT $\rightarrow(\operatorname{Precedence}(\operatorname{Data}(\mathrm{D}))>=$ Precedence $(\operatorname{Data}(\mathrm{D} 1))))$

Rec_Buffer(Receiver) BECOMES Rec_Buffer'(Receiver) SET_DIFF \{D\} \& IF Well_Formed(D) THEN Action Items(Receiver) BECOMES

FI Action_Items'(Receiver) UNION (D\}

END Top_Level 
LEVEL Second_Level REFINES Top_Level

TYPE

DataGram,

Message,

Location,

Message_Type,

Location_Type,

Time,

DataGrams IS SET OF DataGram,

Locations IS SET OF Location,

Precedence_Level IS TYPEDEF T:Integer( $\mathrm{T}>=1 \& \mathrm{~T}<=3)$,

Link,

Links IS SET OF Link

\section{CONSTANT}

TO(DataGram): Location,

FROM(DataGram): Location,

Data(DataGram): Message,

Timestamp(DataGram): Time,

Precedence(Message): Precedence Level,

Type_of_Message(Message): Message_Type,

Type_of_Site(Location): Location_Type.

Consistent_Message(Message_Type,Location_Type): Boolean,

Well_Formed(DataGram): Boolean,

Next_Hop(Location,Location):Location,

Net_Ärch:Links,

Link__OK(Location,Location,Links):Boolean

\section{VARIABLE}

Send_Buffer(Location):DataGrams,

Rec_Buffer(Location):DataGrams,

Trans_Buffer(Location):DataGrams,

Action_Items(Location):DataGrams,

Now:Time,

Operational_Links:Links

\section{INITIAL}

FORALL L:Location (

Send_Buffer $(L)=$ Empty

\& $\operatorname{Rec} B u f f e r(L)=$ Empty

\& Trans_Buffer $(\mathrm{L})=$ Empty

$\&$ Operational_Links $=$ Net_Arch) 
TRANSITION Send ( Sender:Location, Receivers:Locations, M:Message ) ENTRY EXIT

Consistent_Message(Type_of_Message(M), Type_of_Site(Sender))

Send Buffer(Sender) BECOMES Send_Buffer'(Sender) UNION

[SETDEF D:DataGram

EXISTS R:Location (

$R$ ISIN Receivers

$\& T O(D)=R$

$\&$ FROM(D) $=$ Sender

$\& \operatorname{Data}(D)=M$

\& Timestamp(D) $=$ Now $) \mid$

TRANSITION Intermediate ( D:DataGram )

ENTRY

EXISTS L:Location(

(D ISIN Trans_Buffer(L) |D ISIN Send_Buffer(L))

\& Next_Hop(TO'D $(D), L)^{-}=$TO(D)

\& Link_OK(L,Next_Hop(TO(D),L),Operational_Links)

\& FORALL D1:DataGram, L1:Location (

( (D1 ISIN Trans Buffer(L1) |D1 ISIN Send Buffer(L1)) \& Link_OK(L1,Next_Hop(TO(D1),L1),Operational_Links) )

$\rightarrow(\operatorname{Precedence}(\operatorname{Data}(\mathrm{D}))>=$ Precedence $(\operatorname{Data}(\mathrm{D} 1) \overline{)})))$

EXIT

UNIQUE L:Location( (D ISIN Trans_Buffer'(L)|D ISIN Send_Buffer'(L))

\& Send_Buffer(L) BECOMĒS Send_Buffer'(L) SET_DIFF (D)

$\&$ Trans_Buffer(L) BECOMES Trans_Buffer'(L) SET_DIFF (D)

\& Trans Buffer(Next Hop(TO(D),L)) BECOMES

Trans_Buffer'(Next_Hop(TO(D),L)) UNION (D\})

TRANSITION Final ( D:DataGram )

ENTRY

EXISTS L:Location(

(D ISIN Trans_Buffer(L) | D ISIN Send_Buffer(L))

$\&$ Next_Hop(TO(D),L)=TO(D)

\& Link_OK(L,Next_Hop(TO(D),L),Operational_Links)

\& FORALL D1:DataGram, L1:Location (

( (DI ISIN Trans Buffer(L1) |DI ISIN Send Buffer(L1)) \& Link_OK(L1,Next_Hop(TO(D1),L1),Operational_Links)) $\rightarrow\left(\operatorname{Precedence}(\operatorname{Data}(\mathrm{D}))>=\right.$ Precedence $\left.\left.\left.(\operatorname{Data}(\mathrm{Dl}))^{-}\right)\right)\right)$

EXIT

UNIQUE L:Location( (D ISIN Trans_Buffer'(L) | D ISIN Send Buffer'(L))

\& Send_Buffer(L) BECOMES Send_Buffer'(L) SET_DIFF $\{D\}$

\& Trans_Buffer(L) BECOMES Trans_Buffer'(L) SET DIFF (D)

$\&$ Rec_Buffer(TO(D)) BECOMES Rec_Buffer'(TO(D)) UNION (D))

TRANSITION Receive (Receiver:Location, D:DataGram)

ENTRY

D ISIN Rec Buffer(Receiver)

\& FORALL D $1:$ DataGram (DI ISIN Rec Buffer(Receiver)

$\rightarrow(\operatorname{Precedence}(\operatorname{Data}(\mathrm{D}))>=\operatorname{Precedence}(\operatorname{Data}(\mathrm{D} 1))))$ 
EXIT

Rec_Buffer(Receiver) BECOMES Rec_Buffer'(Receiver) SET_DIFF $\{D\}$

\& IF Well_Formed(D)

THEN Action_ltems(Receiver) BECOMES

FI Action_Items'(Receiver) UNION (D)

TRANSITION Remove_Links(L: Links)

ENTRY

Operational_Links $=$ Operational_Links' SET_DIFF $\{L\}$

TRANSITION Restore_Links(L: Links)

ENTRY

L CONTAINED_IN Net_Arch

EXIT

Operational_Links = Operational_Links' UNION $\{L\}$

\section{IMPLEMENTATION}

DataGram $==$ DataGram,

Message $==$ Message,

Location = Location,

Message_Type $=$ Message_Type,

Location_Type $=$ Location_Type,

Time $==$ Time,

DataGrams $=$ DataGrams,

Locations $==$ Locations,

Precedence_Level $=$ Precedence_Level,

$\mathrm{TO}(\mathrm{D})=\mathrm{TO}(\mathrm{D})$,

$\operatorname{FROM}(D)==F R O M(D)$,

$\operatorname{Data}(D)==\operatorname{Data}(D)$,

Timestamp(D) $=$ Timestamp(D),

Precedence $(M)=$ Precedence $(M)$.

Type_of_Message $(M)=$ Type_of_Message $(M)$,

Type_of_Site $(\mathrm{L})=$ Type_of Site $(\bar{L})$,

Consistent_Message $(M, L)=$ Consistent_Message $(M, L)$,

Well Formed(D) $=$ Well_Formed(D),

Neigh̄bor(L1,L2) $=$ Next_Hop $(L 1, L 2)=L 2$,

Send_Buffer $(L)==$ Send_Buffer $(L)$,

Rec_Buffer $(\mathrm{L})==$ Rec_Buffer(L),

Network $==$ (SETDEF D:Datagram EXISTS L:Location (D ISIN Trans_Buffer(L) ) \},

Action_Items $(\mathrm{L})=$ Action_Items $(\mathrm{L})$,

Now $=$ Now,

$\operatorname{Send}(S, R, M)=\operatorname{Send}(S, R, M)$,

Transport(D) $==$ Final(D) $\mid$ Intermediate(D),

Receive $(S, D)==\operatorname{Receive}(S, D)$

END Second Level

END COMMUNICATIONS 

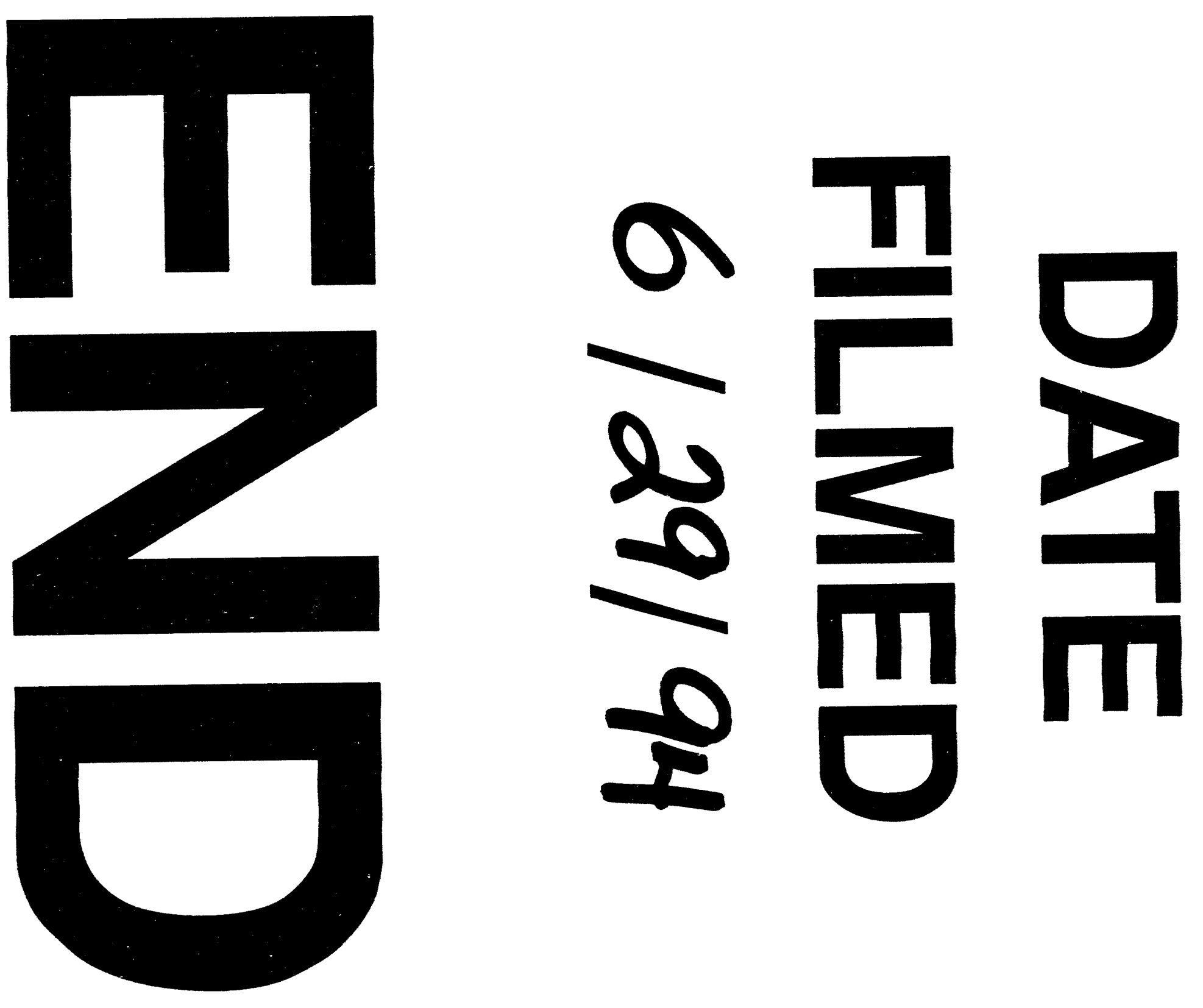

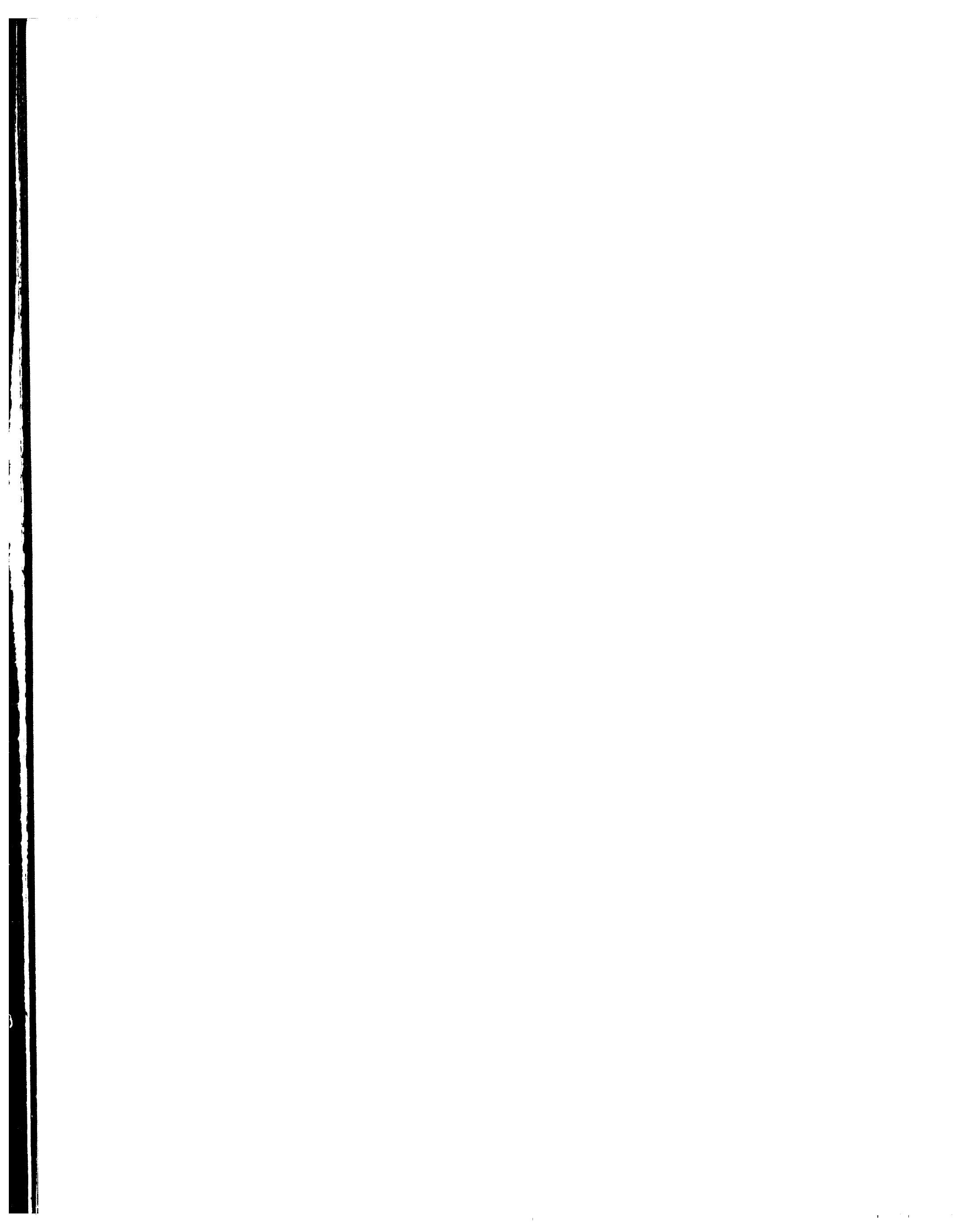